\title{
Impact factors can mislead
}

SIR - Impact factors (IFs) for scientific journals, developed by the Institute for Scientific Information (ISI) and published in the section "Journals per category, ranked by Impact Factor" of the Journal Citation Reports (JCR), are frequently used to evaluate the status of scientific journals or even the publication output of scientists. The IF of a journal in year $T$ is defined as the number of citations in year $T$ to documents published in that journal in years $T-1$ and $T-2$, divided by the number of

purchased from ISI. In each category we compared the ranking of journals by IF as printed in the JCR to the one based on our correct IF, by calculating the number of journals moving at least $1,3,5$ or 10 positions. The table shows the five categories affected most severely, measured through the percentage of journals moving at least one position in the ranking. The categories listed relate not only to the medical sciences but also to chemistry and engineering sciences. The percentage of journals drop-

FIVE CATEGORIES SHOWING LARGEST DIFFERENCES IN RANKINGS BY IF PRINTED IN ISI'S JCR AND BY CORRECT IMPACT FACTOR

\begin{tabular}{lrrrrr} 
Category & No. & \multicolumn{5}{c}{ \% Journals with rank difference } \\
& journals & $\geq 1$ & $\geq 3$ & $\geq 5$ & $\geq 10$ \\
& 130 & 89.2 & 40.8 & 6.9 & 4.6 \\
$\begin{array}{l}\text { Chemistry } \\
\begin{array}{l}\text { Medicine, general } \\
\quad \text { and internal }\end{array}\end{array}$ & 152 & 80.3 & 32.2 & 11.2 & 3.9 \\
$\begin{array}{c}\text { Pharmacology } \\
\text { and pharmacy }\end{array}$ & 171 & 78.4 & 25.7 & 8.2 & 3.5 \\
$\begin{array}{c}\text { Medicine, research } \\
\quad \text { and experimental }\end{array}$ & 86 & 76.7 & 14.0 & 7.0 & 2.3 \\
$\begin{array}{l}\text { Engineering, electrical } \\
\text { and electronic }\end{array}$ & 114 & 70.2 & 18.4 & 6.1 & 3.5 \\
\hline
\end{tabular}

ping by at least 10 positions exceeds 3.5 in all categories except one. Among these, there is a substantial number of 'top' journals.

Our analyses suggest that journal editors or scientific publishers could, in principle, artificially raise the IFs of their journals. To put it bluntly, if a scientific publisher succeeds in publishing important review articles

citeable documents published in that journal in years $T-1$ and $T-2$. But the concept of citeable document is not defined accurately by ISI.

Eugene Garfield has pointed out that, for 40 leading medical periodicals, journals differ with respect to the numbers and types of documents they publish, and variations exist in impact for different types ${ }^{1}$. We obtained evidence that the IFs of many journals included in the Science Citation Index $(S C I)$ are inaccurate because of an inappropriate definition of citeable documents ${ }^{2}$.

ISI classifies documents into types. In calculating the nominator of the IF, ISI counts citations to all types of documents, whereas as citeable documents in the denominator ISI includes as a standard only normal articles, notes and reviews. However, editorials, letters and several other types are cited rather frequently in a number of journals. When they are cited, these types do contribute to the citation counts in the IF's numerator, but are not included in the denominator. In a sense, the citations to these documents are 'for free'. For instance, taking into account only citations to normal articles, notes and reviews in The Lancet, the 'correct' impact factor of this journal in 1992 would be 43 per cent lower than the IF listed in the $J C R$.

We calculated for each $S C I$ journal a 'correct' IF for the year 1994 by taking into account in the IF's numerator only citations to articles, notes and reviews, and including in the denominator the same three types. We used a special datafile as an editorial, or including a lively correspondence section, the IF of his or her journal may go up substantially. Moreover, scientists whose publication output is weighted by the IFs printed in the $J C R$ may see their scores descend when correct IFs are used.

Evaluators of scientific output or journal performance should be cautious in using the impact factors printed in ISI's Journal Citation Reports.

\section{H. F. Moed}

Th. N. van Leeuwen

Centre for Science

and Technology Studies,

Leiden University,

2300 RB Leiden,

The Netherlands

1. Garfield, E. Current Contents, 12 January 1987

2. Moed, H. F. \& van Leeuwen, Th. N. J. Am. Soc. Inf. Sci. 46, 461-467 (1995).

\section{Failed professor}

SIR - Your journal has shown itself sensitive about Spanish scientific policy and assessment of and awards to university professors and researchers.

The present system of marking and assessment, based only on the Science Citation Index $(S C I)$ of the Journal Citation Reports, is unfair to those who have contributed to international journals but apparently not the right ones.

Nobody doing research in Spain at the beginning of the 1980 s could foresee that a list of journals compiled by the $S C I$ was going to determine whether one's research was 'valid' or not. Research productivity is now evaluated by a National Committee selected by the Spanish Science and Education Ministry, which simply rates one's research according to the number of $S C I$ publications accumulated over a sixyear period.

This may work in some fields, but it fails in botany, geology and zoology, for example, because these disciplines do not generate as many articles or citations as, say, biotechnology or genetics. The combination of less frequent citations and a publishing market now saturated with new journals renders the $S C I$ estimated impact factor of these publications close to zero. Many titles are never indexed by the $S C I$.

I am a graduate zoologist, doctor and university professor who was failed by a National Committee which, it seems, takes account only of the $S C I$ ratings. It is ironic never to have failed an examination as an undergraduate, to have been awarded a doctorate in zoology and to have obtained a professorship and then to have been failed by a National Committee. Despite a research track record judged by editorial boards, granting agencies and international colleagues to have been active and productive, I was graded 4 on a scale of 10 .

There is considerable room for improvement in the way the system operates.

José I. Saiz-Salinas

Dpto Biología Animal y Genética,

Universidad del País Vasco,

48.080 Bilbao, Spain

\section{Slow, slow...}

SIR - You perpetuate the myth that HTTP is inherently slower than FTP for single file transfers (Nature 380, 380; 1996). This is patently untrue; both protocols relegate the responsibility for file transfer to a lower level protocol, TCP (transmission control protocol), identical in both cases. In fact, HTTP is marginally more efficiently designed than FTP as it transfers the file down the same connection as the request, whereas FTP opens an entirely new connection for the file transfer.

So, if it can be experimentally verified that an HTTP is indeed slower than FTP (a fact often claimed but rarely supported by any evidence) one must conclude that the difference is caused by poor implementation of the protocol, either by the client or the server, or both.

Of course, HTTP is poorly designed in other ways, particularly when used to transfer modern Web pages made up from many files, as the article correctly points out.

\section{Ben Laurie}

A. L. Digital Ltd,

5 Fairlawn Grove,

London W4 5EL, UK 